\title{
On trigonometric sums with random frequencies
}

\author{
Alina Bazarova ${ }^{1}$, István Berkes ${ }^{2}$ and Marko Raseta ${ }^{3}$
}

\begin{abstract}
We prove that if $I_{k}$ are disjoint blocks of positive integers and $n_{k}$ are independent random variables with uniform distribution on $I_{k}$, then

$$
N^{-1 / 2} \sum_{k=1}^{N}\left(\sin 2 \pi n_{k} x-\mathbb{E}\left(\sin 2 \pi n_{k} x\right)\right)
$$

has, with probability 1, a mixed Gaussian limit distribution relative to the interval $(0,1)$ equipped with Lebesgue measure. We also investigate the case when $n_{k}$ have continuous uniform distribution on disjoint intervals $I_{k}$ on the positive axis.
\end{abstract}

\section{Introduction}

Salem and Zygmund [7] proved that if $\left(n_{k}\right)$ is a sequence of positive integers satisfying the Hadamard gap condition

$$
n_{k+1} / n_{k} \geq q>1 \quad(k=1,2, \ldots)
$$

then the sequence $\sin 2 \pi n_{k} x, k \geq 1$ obeys the central limit theorem, i.e.

$$
N^{-1 / 2} \sum_{k=1}^{N} \sin 2 \pi n_{k} x \stackrel{d}{\longrightarrow} N(0,1 / 2)
$$

with respect the the probability space $(0,1)$ equipped with Borel sets and Lebesgue measure. Here the exponential growth condition (1.1) can be weakened, but as Erdôs

1) Warwick Biology Centre, University of Warwick, GB. Email: a. bazarova@warwick.ac.uk.

2) Alfréd Rényi Institute of Mathematics, Reáltanoda u. 13-15, 1053 Budapest, Hungary. Email: berkes.istvan@renyi.mta.hu. Research supported by Austrian Science Fund (FWF) Grant P24302-N18 and NKFIH Grant K 108615.

3) Department of Mathematics, Keele University, GB. Email: m.raseta@keele.ac.uk. 
[3] showed, there exists a sequence $\left(n_{k}\right)$ growing faster than $e^{\sqrt{k}}$ such that the CLT (1.2) fails. On the other hand, using random constructions one can find slowly growing sequences $\left(n_{k}\right)$ satisfying (1.2). Salem and Zygmund [8] proved that if $\xi_{1}, \xi_{2}, \ldots$ are independent random variables on some probability space $(\Omega, \mathcal{F}, \mathbb{P})$ taking the values 0 and 1 with probability $1 / 2-1 / 2$ and $\left(n_{k}\right)$ denotes the set of indices $j$ such that $\xi_{j}=1$, then with $\mathbb{P}$-probability 1 , the CLT (1.2) holds. For this sequence $\left(n_{k}\right)$ we have $n_{k} \sim 2 k$ and by the theorem of "pure heads" we have $n_{k+1}-n_{k}=O(\log k)$. Berkes [1] showed that if $\mathbb{N}=\cup_{k=1}^{\infty} I_{k}$ where $I_{1}, I_{2}, \ldots$ are disjoint intervals of positive integers such that $\left|I_{k}\right| \rightarrow \infty$, and $n_{1}, n_{2}, \ldots$ are independent random variables on some probability space $(\Omega, \mathcal{F}, \mathbb{P})$ such that $n_{k}$ is uniformly distributed on $I_{k}$, then with $\mathbb{P}$-probability 1 , $\sin 2 \pi n_{k} x$ satisfies the CLT (1.2). Thus, given any positive sequence $\omega_{k} \rightarrow \infty$, there exists an increasing sequence $\left(n_{k}\right)$ of positive integers such that $n_{k+1}-n_{k}=O\left(\omega_{k}\right)$ and $\sin 2 \pi n_{k} x$ satisfies (1.2). In [1] the question was raised if the CLT (1.2) can hold for any sequence $\left(n_{k}\right)$ with $n_{k+1}-n_{k}=O(1)$. Bobkov and Götze [2] showed that the answer to this question is negative, and in particular, if in the construction in [1] we choose $\left|I_{k}\right|=d$ for $k=1,2, \ldots$, then with probability 1 , the limit distribution of $N^{-1 / 2} \sum_{k=1}^{N} \sin 2 \pi n_{k} x$ is mixed normal. On the other hand, Fukuyama [4] showed, using another type of random construction, that for any $0<\sigma^{2}<1 / 2$ there exists a sequence $\left(n_{k}\right)$ of integers with bounded gaps $n_{k+1}-n_{k}$ such that (1.2) holds with a limiting normal distribution with variance $\sigma^{2}$. The purpose of the present paper is to return to the random models in [1], [2] and investigate the case of constant block sizes $\left|I_{k}\right|=d$, allowing arbitrary gaps between the blocks. We will prove the following result.

Theorem 1. Let $I_{1}, I_{2}, \ldots$ be disjoint blocks of consecutive positive integers with size d and let $n_{1}, n_{2}, \ldots$ be a sequence of independent random variables on a probability space $(\Omega, \mathcal{A}, \mathbb{P})$ such that $n_{k}$ is uniformly distributed over $I_{k}$. Let $\lambda_{k}(x)=\mathbb{E}\left(\sin 2 \pi n_{k} x\right)$. Then $\mathbb{P}$-almost surely

$$
\frac{1}{\sqrt{N}} \sum_{k=1}^{N}\left(\sin 2 \pi n_{k} x-\lambda_{k}(x)\right) \stackrel{d}{\longrightarrow} N(0, g)
$$

over the probability space $((0,1), \mathcal{B}, \lambda)$, where $\mathcal{B}$ is the Borel $\sigma$-algebra in $(0,1), \lambda$ is the Lebesgue measure,

$$
g(x)=\frac{1}{2}\left(1-\frac{\sin ^{2} d \pi x}{d^{2} \sin ^{2} \pi x}\right)
$$

and $N(0, g)$ denotes the distribution with characteristic function $\int_{0}^{1} e^{-g(x) t^{2} / 2} d x$.

Here $g \geq 0$ and $N(0, g)$ is the distribution of $\sqrt{g} \zeta$, where $\zeta$ is a standard normal random variable on $(0,1)$, independent of $g$. Clearly, $N(0, g)$ is a variance mixture of zero mean Gaussian distributions.

Note that $\sum_{k=1}^{N} \lambda_{k}(x)=\mathbb{E}\left(\sum_{k=1}^{N} \sin 2 \pi n_{k} x\right)$ is the averaged version of $\sum_{k=1}^{N} \sin 2 \pi n_{k} x$, a nonrandom trigonometric sum and Theorem 1 states that the fluctuations of the 
random trigonometric sum $\sum_{k=1}^{N} \sin 2 \pi n_{k} x$ around its nonrandom average part always have a mixed normal limit distribution. If $\cup_{k=1}^{\infty}\left|I_{k}\right|=\mathbb{N}$, i.e. there are no gaps between the blocks $I_{k}$, then $\sum_{k=1}^{n} \lambda_{k}(x)=O(1)$ for any fixed $x$ and thus (1.3) holds without the $\lambda_{k}(x)$, yielding the result of Bobkov and Götze [2]. Letting $\Delta_{k}$ denote the number of integers between $I_{k}$ and $I_{k+1}$ (the "gaps"), we will see that the CLT (1.3) also holds with $\lambda_{k}(x)=0$ if $\Delta_{k}$ is nondecreasing and $\Delta_{k}=O\left(k^{\gamma}\right)$ for some $\gamma<1 / 4$. If $\Delta_{k}$ grows at least exponentially, then so does the sequence $\left(A_{k}\right)$, where $A_{k}$ denotes the smallest integer of $I_{k}$. Now

$$
\lambda_{k}(x)=\frac{\sin d \pi x}{d \sin \pi x} \sin 2 \pi\left(A_{k}+d / 2-1 / 2\right) x
$$

and from the CLT of Salem and Zygmund [7] it follows that the limit distribution of $N^{-1 / 2} \sum_{k=1}^{N} \lambda_{k}(x)$ is $N\left(0, g^{*}\right)$, where

$$
g^{*}(x)=\frac{\sin ^{2} d \pi x}{2 d^{2} \sin ^{2} \pi x}
$$

By Theorem 1, the limit distribution of $N^{-1 / 2} \sum_{k=1}^{N}\left(\sin 2 \pi n_{k} x-\lambda_{k}(x)\right)$ is $N(0, g)$ with $g$ in (1.4) and the convolution of these two mixed Gaussian laws is $N(0,1 / 2)$, which is exactly the limit distribution of $N^{-1 / 2} \sum_{k=1}^{N} \sin 2 \pi n_{k} x$ by the theorem of Salem and Zygmund, since $\left(n_{k}\right)$ grows exponentially. Thus the pure Gaussian limit distribution of $N^{-1 / 2} \sum_{k=1}^{N} \sin 2 \pi n_{k} x$ is obtained as the combination of two mixed Gaussian distributions $N(0, g)$ with $g$ in (1.4) and $N\left(0, g^{*}\right)$ with $g^{*}$ in (1.6).

It is worth noting that for any fixed $x \in(0,1), \sin 2 \pi n_{k} x-\lambda_{k}(x)$ are independent, uniformly bounded mean zero random variables on $(\Omega, \mathcal{A}, \mathbb{P})$ and

$$
\begin{aligned}
& \mathbb{E}\left(\sin 2 \pi n_{k} x-\lambda_{k}(x)\right)^{2}=\mathbb{E}\left(\sin ^{2} 2 \pi n_{k} x\right)-\lambda_{k}^{2}(x) \\
& =\frac{1}{d} \sum_{j \in I_{k}} \sin ^{2} 2 \pi j x-\left(\frac{1}{d} \sum_{j \in I_{k}} \sin 2 \pi j x\right)^{2}=g(x)
\end{aligned}
$$

by elementary calculations. Thus by the law of the iterated logarithm we have for any fixed $x \in(0,1)$ with $\mathbb{P}$-probability 1

$$
\limsup _{N \rightarrow \infty} \frac{1}{\sqrt{2 N \log \log N}} \sum_{k=1}^{N}\left(\sin 2 \pi n_{k} x-\lambda_{k}(x)\right)=\sqrt{g(x)} .
$$

By Fubini's theorem, with $\mathbb{P}$-probability 1 relation (1.7) holds for almost every $x \in$ $(0,1)$ with respect to Lebesgue measure, yielding the LIL corresponding to (1.3). Actually, the previous argument also shows that for any fixed $x \in(0,1)$ we have $(1.3)$ over the probability space $(\Omega, \mathbb{A}, \mathbb{P})$, with $N(0, g)$ replaced by $N(0, g(x))$. However, Fubini's theorem does not work for distributional results and thus we cannot interchange the role of $x \in(0,1)$ and $\omega \in \Omega$ and we will need an elaborate argument in Section 2 to prove Theorem 1 .

Formula (1.4) shows that for any $0<x<1$ we have $\lim _{x \rightarrow \infty} g(x)=1 / 2$ and thus for large $d$ the sequence $\sin 2 \pi n_{k} x-\lambda_{k}(x)$ nearly satisfies the ordinary CLT and LIL 
with limit distribution $N(0,1 / 2)$ and limsup $=1 / 2$, just as lacunary trigonometric series with exponential gaps. Formally, this is not surprising since for large $d$ the expected gaps $\mathbb{E}\left(n_{k+1}-n_{k}\right)$ in our sequence are large. As the pictures of $g$ for $d=3$ and $d=10$ below show, however, the near CLT and LIL actually hold for relatively small values of $d$ such as $d=10$. Thus the reason of the near CLT and LIL is not solely large gaps in the the sequence $\left(n_{k}\right)$ but the random fluctuations of the sequence $\left(n_{k}\right)$ as well.
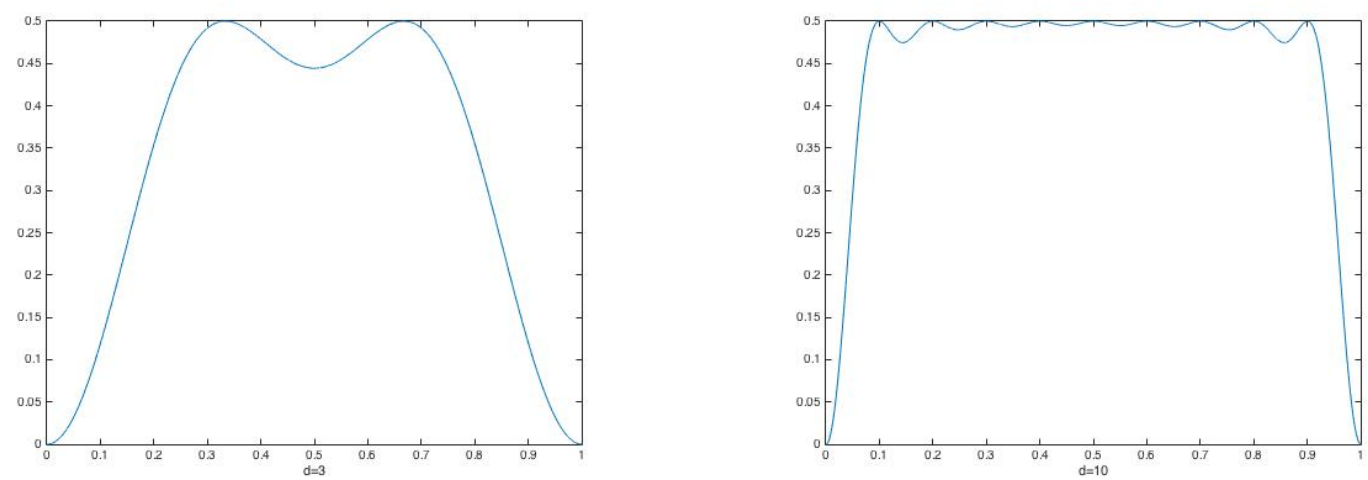

The analogue of Theorem 1 is valid also in the case when $n_{1}, n_{2}, \ldots$ have continuous uniform distribution over the intervals $I_{1}, I_{2}, \ldots$ To formulate the result, define the probability measure $\mu$ on the Borel sets of $\mathbb{R}$ by

$$
\mu(A)=\frac{1}{\pi} \int_{A}\left(\frac{\sin x}{x}\right)^{2} d x, \quad A \subset \mathbb{R} .
$$

Theorem 2. Let $n_{1}, n_{2}, \ldots$ be a sequence of independent random variables on a probability space $(\Omega, \mathcal{A}, \mathbb{P})$ such that $n_{k}$ has continuous uniform distribution on the interval $\left[A_{k}, A_{k}+B\right]$, where $A_{k+1}-A_{k} \geq B+2, k=1,2, \ldots$ Let $\lambda_{k}(x)=\mathbb{E}\left(\sin n_{k} x\right)$. Then $\mathbb{P}$-almost surely

$$
\frac{1}{\sqrt{N}} \sum_{k=1}^{N}\left(\sin n_{k} x-\lambda_{k}(x)\right) \stackrel{d}{\longrightarrow} F
$$

with respect to the probability space $(\mathbb{R}, \mathcal{B}, \mu)$, where the characteristic function of $F$ is

$$
\phi(\lambda)=\int_{-\infty}^{+\infty} \exp \left(-\frac{\lambda^{2}}{4}\left(1-\frac{4 \sin ^{2}(B x / 2)}{B^{2} x^{2}}\right)\right) d \mu(x) .
$$

\section{Proofs}

We will give the proof of Theorem 2, where the calculations are slightly simpler. Let

$$
\varphi_{k}(x)=\sin n_{k} x-\mathbb{E}\left(\sin n_{k} x\right)
$$


and

$$
T_{N}=\frac{1}{\sqrt{N}} \sum_{k=1}^{N} \varphi_{k}(x) .
$$

By $A_{k+1}-A_{k} \geq B+2$ and the fact that

$$
\int_{-\infty}^{+\infty} \cos \alpha x\left(\frac{\sin x}{x}\right)^{2} d x=0 \text { for }|\alpha|>2
$$

(see e.g. Hartman [5]) it follows that for every fixed $\omega \in \Omega$ the functions $\varphi_{k}$ are orthogonal over $L_{\mu}^{2}(\mathbb{R})$ and thus elementary algebra shows that the $L_{\mu}^{2}(\mathbb{R})$ norm of $\left|T_{M}-T_{N^{3}}\right|$ is at most $C / \sqrt{N}$ for $N^{3} \leq M \leq(N+1)^{3}$ with an absolute constant $C$. Hence to prove (1.8) it suffices to show that $T_{N^{3}} \stackrel{d}{\longrightarrow} F \mathbb{P}$-a.s.

A simple calculation shows that

$$
\begin{aligned}
& \lambda_{k}(x)=\mathbb{E}\left(\sin n_{k} x\right)=\frac{1}{B} \int_{A_{k}}^{A_{k}+B} \sin t x d t=\frac{1}{B x}\left(\cos A_{k} x-\cos \left(A_{k}+B\right) x\right) \\
& =\frac{2 \sin (B x / 2)}{B x} \sin \left(A_{k}+B / 2\right) x
\end{aligned}
$$

and

$$
\mathbb{E}\left(\cos 2 n_{k} x\right)=\frac{1}{B} \int_{A_{k}}^{A_{k}+B} \cos 2 t x d t=\frac{\sin B x}{B x} \cos \left(2 A_{k}+B\right) x .
$$

Thus

$$
\begin{aligned}
\mathbb{E} \varphi_{k}^{2}(x) & =\mathbb{E}\left(\sin ^{2} n_{k} x\right)-\lambda_{k}^{2}(x)=\frac{1}{2}\left(1-\mathbb{E}\left(\cos 2 n_{k} x\right)\right)-\lambda_{k}^{2}(x) \\
& =\frac{1}{2}-\frac{\sin B x}{2 B x} \cos \left(2 A_{k}+B\right) x-\frac{4 \sin ^{2}(B x / 2)}{B^{2} x^{2}} \sin ^{2}\left(A_{k}+B / 2\right) x \\
& =\left(\frac{1}{2}-\frac{2 \sin ^{2}(B x / 2)}{B^{2} x^{2}}\right)+\left(\frac{2 \sin ^{2}(B x / 2)}{B^{2} x^{2}}-\frac{\sin B x}{2 B x}-\right) \cos \left(2 A_{k}+B\right) x .
\end{aligned}
$$

From (2.10), $A_{k+1}-A_{k} \geq B+2$ and elementary trigonometric identities it follows that the functions $\cos \left(2 A_{k}+B\right) x$ are orthogonal in $L_{\mu}^{2}(\mathbb{R})$ and thus the RademacherMenshov convergence theorem implies that $\sum_{k=1}^{\infty} k^{-1} \cos \left(2 A_{k}+B\right) x$ converges $\mu$ almost everywhere. Consequently, the Kronecker lemma implies

$$
\lim _{N \rightarrow \infty} \frac{1}{N} \sum_{k=1}^{N} \cos \left(2 A_{k}+B\right) x=0 \quad \mu-\text { a.e. }
$$

and thus

$$
\lim _{N \rightarrow \infty} \frac{1}{N} \sum_{k=1}^{N} \mathbb{E} \varphi_{k}^{2}(x)=\frac{1}{2}\left(1-\frac{4 \sin ^{2}(B x / 2)}{B^{2} x^{2}}\right) \quad \mu \text { - a.e. }
$$

Since for fixed $x \varphi_{k}^{2}(x)-\mathbb{E} \varphi_{k}^{2}(x), k=1,2, \ldots$ are independent, uniformly bounded, zero mean random variables, the strong law of large numbers yields

$$
\lim _{N \rightarrow \infty} \frac{1}{N} \sum_{k=1}^{N}\left(\varphi_{k}^{2}(x)-\mathbb{E} \varphi_{k}^{2}(x)\right)=0 \quad \mathbb{P}-\text { a.s. }
$$


and thus we conclude that for $\mu$-a.e. $x$ we have $\mathbb{P}$-almost surely

$$
\lim _{N \rightarrow \infty} \frac{1}{N} \sum_{k=1}^{N} \varphi_{k}^{2}(x)=\frac{1}{2}\left(1-\frac{4 \sin ^{2}(B x / 2)}{B^{2} x^{2}}\right) .
$$

By Fubini's theorem, $\mathbb{P}$-almost surely the last relation holds for $\mu$-almost all $x \in \mathbb{R}$. Fix $\lambda \in \mathbb{R}$. Using $\left|\varphi_{k}(x)\right| \leq 2$ and

$$
\exp (z)=(1+z) \exp \left(\frac{z^{2}}{2}+o\left(z^{2}\right)\right) \quad z \rightarrow 0
$$

we get

$$
\exp \left(\frac{i \lambda}{\sqrt{N}} \varphi_{k}(x)\right)=\left(1+\frac{i \lambda}{\sqrt{N}} \varphi_{k}(x)\right) \exp \left(-\frac{\lambda^{2} \varphi_{k}^{2}(x)}{2 N}+o\left(\frac{\lambda^{2} \varphi_{k}^{2}(x)}{N}\right)\right)
$$

as $N \rightarrow \infty$, uniformly in $x$ and the implicit variable $\omega \in \Omega$. Thus the characteristic function

$$
\phi_{T_{N}}(\lambda)=\int_{-\infty}^{\infty} \exp \left(\frac{i \lambda}{\sqrt{N}} \sum_{k=1}^{N} \varphi_{k}(x)\right) d \mu(x)=\int_{-\infty}^{\infty} \exp \left(\frac{i \lambda}{\sqrt{N}} \sum_{k=1}^{N} \varphi_{k}(x, \omega)\right) d \mu(x)
$$

of $T_{N}$ with respect to the probability space $(\mathbb{R}, \mathcal{B}, \mu)$ can be written as

$$
\begin{aligned}
\phi_{T_{N}}(\lambda)= & \int_{-\infty}^{+\infty} \prod_{k=1}^{N}\left(1+\frac{i \lambda}{\sqrt{N}} \varphi_{k}(x)\right) \\
& \times \exp \left(-(1+o(1)) \frac{\lambda^{2}}{2 N} \sum_{k=1}^{N} \varphi_{k}^{2}(x)\right) \frac{1}{\pi}\left(\frac{\sin x}{x}\right)^{2} d x .
\end{aligned}
$$

For simplicity let

$$
\hat{g}(x)=\frac{1}{2}\left(1-\frac{4 \sin ^{2}(B x / 2)}{B^{2} x^{2}}\right) .
$$

Using $1+x \leq e^{x}$ and $\left|\varphi_{k}(x)\right| \leq 2$ we get

$$
\begin{aligned}
& \left|\prod_{k=1}^{N}\left(1+\frac{i \lambda}{\sqrt{N}} \varphi_{k}(x)\right)\right|=\prod_{k=1}^{N}\left(1+\frac{\lambda^{2}}{N} \varphi_{k}^{2}(x)\right)^{1 / 2} \\
& \leq \exp \left(\frac{\lambda^{2}}{2 N} \sum_{k=1}^{N} \varphi_{k}^{2}(x)\right) \leq e^{2 \lambda^{2}}
\end{aligned}
$$

and thus the dominated convergence theorem and (2.12) imply $\mathbb{P}$-almost surely

$$
\phi_{T_{N}}(\lambda)=\int_{-\infty}^{+\infty} \prod_{k=1}^{N}\left(1+\frac{i \lambda}{\sqrt{N}} \varphi_{k}(x)\right) \exp \left(-\lambda^{2} \hat{g}(x) / 2\right) \frac{1}{\pi}\left(\frac{\sin x}{x}\right)^{2} d x+o(1) .
$$


Since the characteristic function $\phi(\lambda)$ of $F$ in (1.8) is given by (1.9), to prove that $T_{N^{3}} \stackrel{d}{\longrightarrow} F \mathbb{P}$-a.s., it remains to show that letting

$$
\Gamma_{N}=\int_{-\infty}^{+\infty}\left[\prod_{k=1}^{N}\left(1+\frac{i \lambda}{\sqrt{N}} \varphi_{k}(x)\right)-1\right] \exp \left(-\lambda^{2} g(x) / 2\right) \frac{1}{\pi}\left(\frac{\sin x}{x}\right)^{2} d x
$$

we have

$$
\Gamma_{N^{3}} \stackrel{\mathbb{P} \text {-a.s. }}{\longrightarrow} 0 .
$$

Clearly

$$
\begin{aligned}
\mathbb{E}\left|\Gamma_{N}\right|^{2}= & \mathbb{E} \int_{-\infty}^{+\infty} \int_{-\infty}^{+\infty}\left[\prod_{k=1}^{N}\left(1+\frac{i \lambda}{\sqrt{N}} \varphi_{k}(x)\right)-1\right]\left[\prod_{k=1}^{N}\left(1-\frac{i \lambda}{\sqrt{N}} \varphi_{k}(y)\right)-1\right] \\
& \times \exp \left(-\lambda^{2} g(x) / 2\right) \exp \left(-\lambda^{2} g(y) / 2\right) d \mu(x) d \mu(y) .
\end{aligned}
$$

Now using the independence of the $\varphi_{k}$ and $\mathbb{E} \varphi_{k}(x)=\mathbb{E} \varphi_{k}(y)=0$ we get

$$
\begin{aligned}
& \mathbb{E}\left[\prod_{k=1}^{N}\left(1+\frac{i \lambda}{\sqrt{N}} \varphi_{k}(x)\right)-1\right]\left[\prod_{k=1}^{N}\left(1-\frac{i \lambda}{\sqrt{N}} \varphi_{k}(y)\right)-1\right] \\
& =\mathbb{E}\left[\prod_{k=1}^{N}\left(1+\frac{i \lambda}{\sqrt{N}} \varphi_{k}(x)\right)\left(1-\frac{i \lambda}{\sqrt{N}} \varphi_{k}(y)\right)\right]-1 \\
& =\mathbb{E}\left[\prod_{k=1}^{N}\left(1+\frac{i \lambda}{\sqrt{N}} \varphi_{k}(x)-\frac{i \lambda}{\sqrt{N}} \varphi_{k}(y)+\frac{\lambda^{2}}{N} \varphi_{k}(x) \varphi_{k}(y)\right)\right]-1 \\
& =\prod_{k=1}^{N}\left(1+\frac{\lambda^{2}}{N} \Psi_{k}(x, y)\right)-1,
\end{aligned}
$$

where $\Psi_{k}(x, y)=\mathbb{E} \varphi_{k}(x) \varphi_{k}(y)$. Thus interchanging the expectation with the double integral in (2.14) we get

$$
\begin{aligned}
\mathbb{E}\left|\Gamma_{N}\right|^{2}= & \int_{-\infty}^{+\infty} \int_{-\infty}^{+\infty}\left[\prod_{k=1}^{N}\left(1+\frac{\lambda^{2}}{N} \Psi_{k}(x, y)\right)-1\right] \times \\
& \times \exp \left(-\lambda^{2} g(x) / 2-\lambda^{2} g(y) / 2\right) d \mu(x) d \mu(y) \\
& \leq \int_{-\infty}^{+\infty} \int_{-\infty}^{+\infty}\left|\prod_{k=1}^{N}\left(1+\frac{\lambda^{2}}{N} \Psi_{k}(x, y)\right)-1\right| d \mu(x) d \mu(y) .
\end{aligned}
$$

Using $\left|\Psi_{k}(x, y)\right| \leq 4$ and $|\log (1+x)-x| \leq C x^{2}$ for all $|x| \leq 1$ and some constant $C>0$, one deduces for all sufficiently large $N$,

$$
\left|\log \prod_{k=1}^{N}\left(1+\frac{\lambda^{2}}{N} \Psi_{k}(x, y)\right)-\sum_{k=1}^{N} \frac{\lambda^{2}}{N} \Psi_{k}(x, y)\right| \leq \frac{16 C \lambda^{4}}{N} .
$$


Thus letting

$$
G_{N}(x, y):=\sum_{k=1}^{N} \frac{\lambda^{2}}{N} \Psi_{k}(x, y)
$$

we get, using $G_{N}(x, y) \leq 4 \lambda^{2}$, that

$$
\prod_{k=1}^{N}\left(1+\frac{\lambda^{2}}{N} \Psi_{k}(x, y)\right)=\exp \left\{G_{N}(x, y)+O\left(\lambda^{4} / N\right)\right\}=1+O\left(\left|G_{N}(x, y)\right|\right)+O(1 / N) .
$$

Thus

$$
\mathbb{E}\left|\Gamma_{N}\right|^{2} \leq C_{1}\left(\frac{1}{N}+\int_{-\infty}^{+\infty} \int_{-\infty}^{+\infty}\left|G_{N}(x, y)\right| d \mu(x) d \mu(y)\right)
$$

for some constant $C_{1}$. In view of $A_{k+1}-A_{k} \geq B+2$ and (2.10), for any $\lambda_{1} \in$ $\left[A_{k}, A_{k}+B\right], \lambda_{2} \in\left[A_{l}, A_{l}+B\right], k \neq l, \sin \lambda_{1} x$ and $\sin \lambda_{2} x$ are orthogonal in $L_{\mu}^{2}(\mathbb{R})$, which implies that $\varphi_{k}$ and $\varphi_{\ell}$ are also orthogonal in $L_{\mu}^{2}(\mathbb{R})$. Since $\Psi_{k}(x, y) \Psi_{l}(x, y)=$ $\mathbb{E} \varphi_{k}(x) \varphi_{l}(x) \varphi_{k}(y) \varphi_{l}(y)$, it follows that

$$
\int_{-\infty}^{+\infty} \int_{-\infty}^{+\infty} \Psi_{k}(x, y) \Psi_{l}(x, y) d \mu(x) d \mu(y)=0 \quad \text { for } k \neq l
$$

and thus by the Cauchy-Schwarz inequality the last integral in $(2.15)$ is $O\left(N^{-1 / 2}\right)$. Hence $\mathbb{E}\left|\Gamma_{N}\right|^{2}=O\left(N^{-1 / 2}\right)$ and thus $\sum_{N \in \mathbb{N}} \mathbb{E}\left|\Gamma_{N^{3}}\right|^{2}<\infty$, implying $\sum_{N \in \mathbb{N}}\left|\Gamma_{N^{3}}\right|^{2}<\infty$ and $\Gamma_{N^{3}} \rightarrow 0 \mathbb{P}$-a.s., completing the proof of (1.8).

In conclusion we prove the claim made after Theorem 1, namely that if the size of the gaps $\Delta_{k}$ between the blocks $I_{k}$ is nondecreasing and satisfies

$$
\Delta_{k}=O\left(k^{\gamma}\right), \quad \gamma<1 / 4
$$

then

$$
N^{-1 / 2} \sum_{k=1}^{N} \lambda_{k}(x) \longrightarrow 0 \quad \text { a.s. }
$$

and thus (1.3) holds with $\lambda_{k}(x)=0$. Since we proved our main limit theorem in the continuous case of Theorem 2, we prove our claim also in the context of Theorem 2 in which case we also assume that the intervals $\left[A_{k}, A_{k}+B\right]$ have integer endpoints. In view of (2.11) it suffices to show that

$$
N^{-1 / 2} \sum_{k=1}^{N} e^{i A_{k} x} \longrightarrow 0 \quad \text { a.s. }
$$

and here nothing changes if we replace $x$ by $2 \pi x$. In the case of constant $\Delta_{k}$ we have $A_{k}=D k+D^{*}$ for some constants $D>0$ and $D^{*}$ and (2.17) is obvious by an explicit 
computation of the sum. Thus we can assume $\Delta_{k} \uparrow \infty$, and then also $A_{k+1}-A_{k} \uparrow \infty$. Recalling that the $A_{k}$ are integers, let us break the sum $\sum_{k=1}^{N} e^{2 \pi i A_{k} x}$ into subsums

$$
Z_{N, r}=\sum_{k \leq N, A_{k+1}-A_{k}=r} e^{2 \pi i A_{k} x}, \quad r=1,2, \ldots
$$

Clearly $Z_{N, r}$ consists of $M_{r}$ consecutive terms of $\sum_{k=1}^{N} e^{2 \pi i A_{k} x}$ for some $M_{r} \geq 0$ and thus in the case $M_{r} \geq 1$ we have for some integer $P_{r} \geq 0$,

$$
\left|Z_{N, r}\right|=\left|\sum_{j=0}^{M_{r}-1} e^{2 \pi i\left(P_{r}+j r\right) x}\right|=\left|\sum_{j=0}^{M_{r}-1} e^{2 \pi i j r x}\right| \leq \frac{1}{\left|e^{2 \pi i r x}-1\right|} \leq \frac{C}{\langle r x\rangle},
$$

except when $r x$ is an integer, where $C$ is an absolute constant and $\langle t\rangle$ denotes the distance of $t$ from the nearest integer. From a well known result in Diophantine approximation theory (see e.g. Kuipers and Niederreiter [6], Definition 3.3. on p. 121 and Exercise 3.5 on page 130), for every $\varepsilon>0$ and almost all $x$ in the sense of Lebesgue measure we have $\langle n x\rangle \geq c n^{-(1+\varepsilon)}$ for some constant $c=c(x)>0$ and all $n \geq 1$. This shows that $Z_{N, r}=O\left(r^{1+\varepsilon}\right)$ a.e. and since by (2.16) the largest $r$ actually occurring in breaking $\sum_{k=1}^{N} e^{2 \pi i A_{k} x}$ into a sum of $Z_{N, r}$ 's is at most $C_{1} N^{\gamma}$, we have

$$
\left|\sum_{k=1}^{N} e^{2 \pi i A_{k} x}\right| \leq C_{2} \sum_{r \leq C_{1} N^{\gamma}} r^{1+\varepsilon}=o(\sqrt{N}) \quad \text { a.e. }
$$

by $\gamma<1 / 4$, upon choosing $\varepsilon$ small enough.

\section{References}

[1] I. Berkes. A central limit theorem for trigonometric series with small gaps. $Z$. Wahrscheinlichkeitstheorie verw. Gebiete 47 (1979), 157-161.

[2] S. Bobkov and F. Götze, Concentration inequalities and limit theorems for randomized sums. Probab. Theory Related Fields 137 (2007), 49-81.

[3] P. ERdôs, On trigonometric sums with gaps. Magyar Tud. Akad. Mat. Kut. Int. Közl. 7 (1962), 37-42.

[4] K. Fukuyama. A central limit theorem for trigonometric series with bounded gaps. Prob. Theory Rel. Fields 149 (2011), 139-148.

[5] P. Hartman. The divergence of non-harmonic gap series, Duke Math. J. 9 (1942), 404-405.

[6] L. Kuipers and H. Niederreiter. Uniform distribution of sequences. Wiley, New York.

[7] R. Salem and A. Zygmund. On lacunary trigonometric series, Proc. Nat. Acad. Sci. USA 33 (1947), 333-338. 
[8] R. Salem and A. Zygmund, Trigonometric series whose terms have random signs. Acta Math. 91 (1954), 245-301. 